\title{
Experimental Analysis of Four-Wave Mixing in a Semiconductor Optical Amplifier Using Frequency Resolved Optical Gating
}

\author{
B.F. Kennedy ${ }^{a}$, K. Bondarczuk ${ }^{b}$, L.P. Barry ${ }^{b}$ \\ a) Departamento Ingenieria Electrica, Universidad de Santiago, Chile \\ b) School of Electronic Engineering, Dublin City University, Ireland \\ Tel+56277864 17, e-mail: bkennedy@lauca.usach.cl
}

\begin{abstract}
Four-wave mixing was performed in a multi-quantum well semiconductor optical amplifier in order to demultiplex 2 ps pulses from $80 \mathrm{GHz}$ to $10 \mathrm{GHz}$. The demultiplexed pulses were measured using frequency resolved optical gating. This technique allows for full characterization of the pulses in both the time and frequency domains. The measurements confirm that the four-wave mixing conjugate signal preserves the phase of the injected signal. Furthermore, in the time domain the influence of the pulse pedestals present on the injected probe pulses is significantly increased on the output four-wave mixing signals. This effect is attributed to the nonlinear suppression of the device gain.
\end{abstract}

\section{INTRODUCTION}

Currently many research groups are focussing on the anticipated future need for all-optical signal processing techniques for applications in optical networks. Among the most attractive devices which have been put forward is the Semiconductor Optical Amplifier (SOA), due to its high nonlinearities, high gain, small size, integratability and relatively low cost. Several techniques exist to perform all-optical signal processing in SOAs. Among these are Cross-Gain Modulation (XGM), Cross-Phase Modulation (XPM) and Cross-Polarization Modulation (XPolM) [1-3]. Another technique which receives a lot of attention is Four-Wave Mixing (FWM). This is a process whereby two signals, a pump and a probe, are injected into the SOA at a frequency detuning, $\Omega$. Due to third order nonlinearities, the gain of the SOA is modulated leading to the creation of new components in the spectrum. The spacing of the new components is determined by the frequency detuning between the pump and the probe signals [4,5]. An interesting property of the generated FWM component is that the phase of this signal is the conjugate of the phase of the injected probe signal. This phase preservation cannot be achieved using any of the other techniques that may be used for all-optical signal processing with SOA's and has huge potential for use in optical systems. One good example is the possibility to perform wavelength conversion and demultiplexing with Differential Phase-Shift Keying (DPSK) format data in all-optical networks $[6,7]$.

If FWM is to be used in high-speed optical networks operating at line rates of 40,80 and $160 \mathrm{~Gb} / \mathrm{s}$, it is critically important to understand the process of FWM between short picosecond pulses in the SOA. To date much work has focussed on this aspect of FWM, but to the best of the author's knowledge, no experimental results have been presented which show a full intensity and phase characterization of FWM between picosecond pulses. In this paper experimental results are presented for the demultiplexed FWM component generated due to the beating between picosecond pulses in the SOA. The pump signal consisted of 4 ps pulses at a repetition rate of $10 \mathrm{GHz}$, whilst the probe consisted of 2 ps pulses at a repetition rate of $80 \mathrm{GHz}$, resulting in a FWM component demultiplexed to $10 \mathrm{GHz}$. The intensity and phase of both the injected pump and probe pulses were measured. It is shown that the phase is preserved in the FWM component, as is expected from the theory. The intensity profile of the FWM signal is also presented and it is shown that the influence of the weak pedestals present in the wings of the injected pulses is significantly enhanced in the conjugate signal. The effect of the enhanced pedestals has been presented before in the case of a single pulse being amplified in the SOA and was attributed to gain depletion effects [8]. This effect should be taken into consideration if FWM is to be realized for all-optical processing in future all-optical networks.

\section{EXPERIMENTAL SETUP}

The experimental setup is illustrated in Fig. 1. A 2 ps pulses were generated using two actively injected modelocked laser sources. The repetition rate of both signals was $10 \mathrm{GHz}$ and the wavelength of the pump laser was $1550 \mathrm{~nm}$ for each measurement taken. This wavelength is close to the peak gain wavelength of the device under test. The wavelength of the probe laser was $1560 \mathrm{~nm}$, resulting in a FWM component being generated at approximately $1540 \mathrm{~nm}$. This may be described as wavelength down conversion. The probe signal was amplified using an Erbium Doped Fibre Amplifier (EDFA) and injected into a multiplexer in order to multiplex the pulses from 10 to $80 \mathrm{GHz}$. After the multiplexer, the signal was amplified once more and a Band-Pass Filter (BPF) was used in order to remove any Amplified Spontaneous Emission (ASE) introduced by the EDFAs. The probe signal was then coupled with the pump pulses before injection into the SOA device. The pump signal was 
amplified and then filtered by a BPF with a passband of $1 \mathrm{~nm}$. This narrow passband had the effect of broadening the pulsewidth from 2 ps to 4 ps. This broadening allowed for FWM components generated due to the beating between the pedestals of the probe signal and the broadened section of the pump signal to be examined. The average powers of the pump and probe signals at $10 \mathrm{GHz}$ and $80 \mathrm{GHz}$ were $4 \mathrm{dBm}$ and $0.25 \mathrm{dBm}$, respectively. An Optical Delay Line (ODL) was placed in the pump arm of the setup to allow for the optimum overlapping of the two signals to produce the most efficient FWM signal. The SOA under test was a multi-quantum well device biased at $200 \mathrm{~mA}$, with a peak gain of $25 \mathrm{~dB}$. At the output of the SOA, a BPF was used to isolate the FWM component from the output spectrum. The FWM component was then examined using an Optical Spectrum Analyzer (OSA) with a resolution bandwidth of $0.07 \mathrm{~nm}$ and also using second-harmonic generation Frequency Resolved Optical Gating (FROG) [9]. The FROG technique generates a threedimensional spectrogram, which is a time-frequency representation of the pulse. A phase retrieval program is then applied to allow for the electric field of the pulse to be determined, giving the complete temporal and spectral characterization of the pulse [10]. Due to the power requirements of the FROG measurement, an EDFA is used to amplify the pulses before they are input into the FROG measurement system. This gives the pulse a larger signal-to-noise ratio resulting in more accurate retrievals of the electric field of the pulse. The EDFA used was designed for the amplification of 2 ps pulses. Low errors of $\approx 4 \times 10^{-5}$ were recorded for the retrieved pulses, indicating that the retrievals were accurate [9].

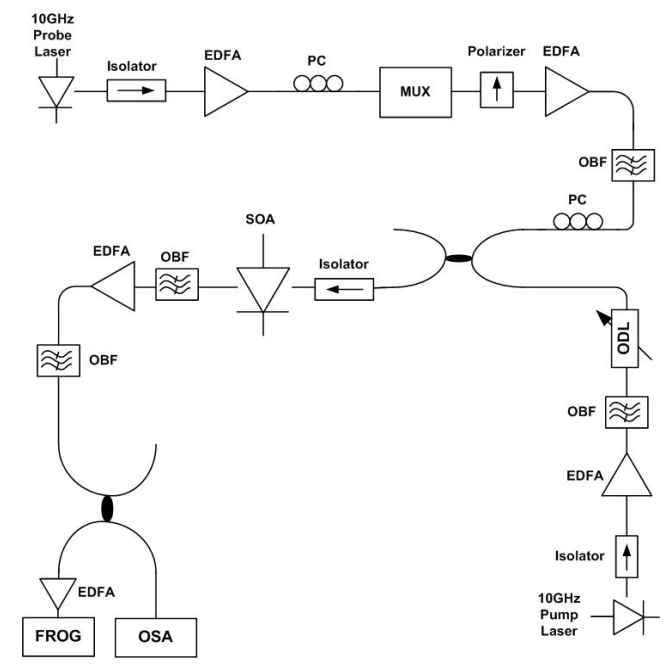

Figure 1. The experimental setup used to perform demultiplexing using FWM in the SOA.

\section{RESULTS}

The intensity of the injected pump and probe signals is shown in Fig. $2 a$. The probe signal has a pulsewidth of 2 ps. The pedestals in the wings of the probe signal are located approximately $40 \mathrm{~dB}$ from the peak of the pulse. These pedestals are introduced in the mode-locking process in the source laser. The broadening introduced by the $1 \mathrm{~nm}$ BPF in the pump arm can be seen in the intensity profile of the pump signal, which has a pulsewidth of 4 ps. The chirp present for both the pump and probe signals is shown in Fig. $2 b$. The linear chirp introduced across the central part of the pulse is very similar for both pump and probe signals. This is to be expected as the pulses are generated in mode-locked laser sources with similar specifications. The slight difference in the slope of the chirp is thought to be due to the BPF present in the pump arm of the setup.
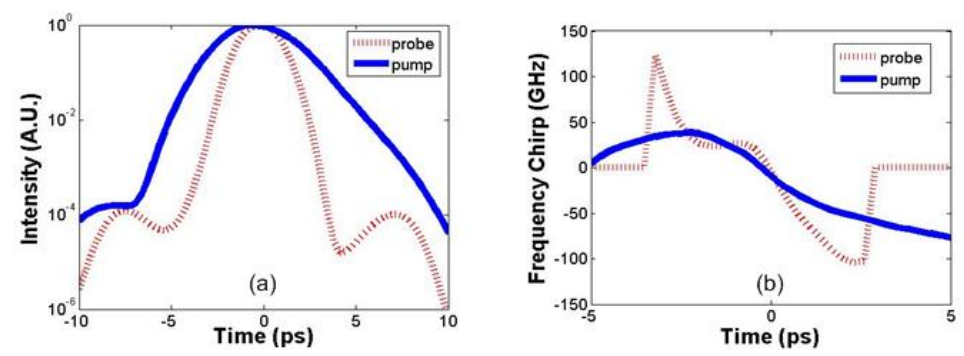

Figure 2. (a) Intensity and (b) chirp of the pump and probe signals injected into the SOA.. 
In Fig. 3 the intensity and chirp of the injected probe signal, FWM component and amplified probe signal after passing through the SOA in co-propagation with the pump are shown. As discussed in relation to Fig. $2 a$, the pedestals which are present on the initial probe pulses are located approximately $40 \mathrm{~dB}$ below the peak of the pulse. After propagation through the SOA the pedestals have increased in significance and are located approximately $20 \mathrm{~dB}$ below the peak of the pulse on the trailing edge. The amplification of the probe pulse pedestals has been explained through the effects of gain saturation [8]. However, in this reference the largest amplification of the pedestals was measured on the leading edge, due to the larger gain seen by this pedestal prior to the depletion of the gain caused by the main pulse. The larger amplification of the trailing edge pedestal in relation to the peak of the pulse, shown in Fig. $3 a$, is thought to be related to the gain-induced refractive index dispersion, which may cause the pump pulses to travel through the SOA at a faster velocity than the probe pulses [11]. Therefore, the leading edge probe pedestals may be amplified in the saturated regime of the SOA gain whilst the trailing edge pedestals may receive a larger gain than both the leading edge pedestals and the main portion of the probe pulse, as the gain will have started to recover.
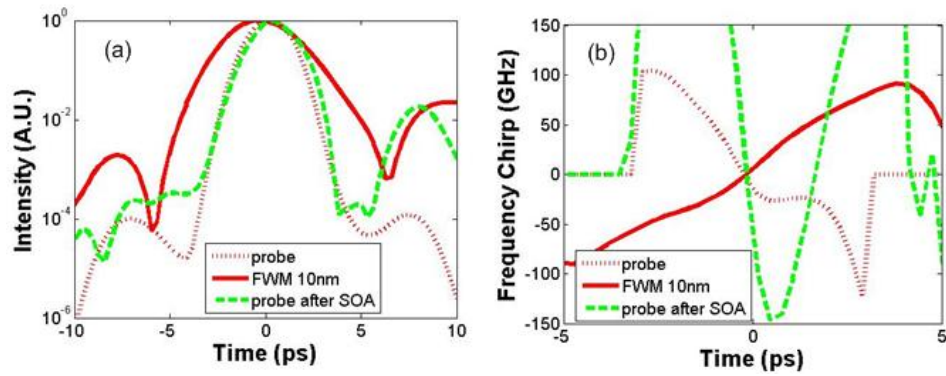

Figure 3. a) Intensity and (b) chirp of the probe, amplified probe and FWM signals at the output of the SOA..

The FWM signal generated at the beat frequency determined by the detuning between the pump and the probe signals is also shown in Fig. 3. Care must be taken in the choice of the detuning in the case when pulses are considered. This detuning must be greater than the spectral width of the pulses under test. However, as the detuning is increased, the efficiency of the FWM process reduces due to the dominance of weaker gain dynamics such as $\mathrm{CH}$ and SHB. In Fig. 3 the intensity and chirp of the FWM signal are shown. The FWM signal is at a lower wavelength, $1540 \mathrm{~nm}$, than the probe signal, therefore the conversion is wavelength down conversion.

From Fig. $3 a$, it can be seen that there is an increase in the influence of the pedestals for the FWM signal in comparison with the amplified probe signal. This is particularly the case for the pulses located on the leading edge of the pulse. The pedestals are located approximately $20 \mathrm{~dB}$ from the peak of the pulse on the trailing edge of the pulse and approximately $27 \mathrm{~dB}$ from the peak of the pulse on the leading edge. This increase is believed to be caused by the combined effects of FWM and SPM in the active region of the device. It may also be seen from Fig. 3 that the FWM signal is considerably wider than the injected and amplified probe signals. This is thought to be primarily due to the two BPFs located at the output of the SOA device, as can be seen in Fig. 1.

The frequency chirp present for the FWM signal, solid line, together with the initial probe frequency chirp, dotted line, is shown in Fig. $3 b$. As the FWM component is the phase conjugate of the injected probe signal, the frequency chirps have opposite slopes. The magnitudes of the chirps are very similar and the FWM component has a linear chirp as does the initial probe signal. It can be seen that no large nonlinear components are introduced to the chirp due to the FWM process. This may be compared to the chirp introduced to the amplified probe signal, dashed line, also shown in Fig. $3 b$. A large nonlinear chirp component is introduced due to SelfPhase Modulation (SPM) in the SOA. The shape of the chirp introduced due to SPM is similar to that which is discussed in the literature [12]. This is another potential advantage of using FWM for all-optical processing in SOA's as compared with other techniques, as the chirp introduced due to SPM is not necessarily transferred to the FWM conjugate signal.

\section{CONCLUSIONS}

Results are presented showing demultiplexing from $80 \mathrm{GHz}$ to $10 \mathrm{GHz}$ based on FWM in a multi-quantum well SOA. Measurements of the converted signal were taken using the FROG measurement technique. This allowed for complete characterization of the FWM signals. The chirp of the converted signal is presented, demonstrating the preservation of the phase. Also, the intensity profile is measured and it is shown that the influence of weak pedestals on the signal injected to the SOA becomes significant after the FWM process. This effect is thought to be related to the fact that the pedestals receive a larger gain than the peak of the pulse due to gain saturation. 
The influence of these pedestals should be taken into consideration when designing future all-optical processing elements based on FWM in SOAs.

\section{REFERENCES}

[1] S. Nakamura, Y. Ueno, and K. Tarima, 168-Gb/s all-optical wavelength converter with a symmetric MachZehnder type switch, IEEE Photon. Tech. Lett., vol. 13, no. 10, pp. 1091-1093, 2001.

[2] G. Contestabile, N. Calabreta, M. Presi, and E. Ciaramella, Single and multicast wavelength conversion at $40 \mathrm{~Gb} / \mathrm{s}$ by means of fast nonlinear polarization switching in an SOA, IEEE Photon. Tech. Lett., vol. 17, pp. 2652-2654, 2005.

[3] B.F. Kennedy, S. Philippe, F. Surre, A.L. Bradley, and P. Landais, Investigation of optimum wavelength converter based on nonlinear polarization rotation in a bulk SOA, IET Optoelectronics, vol. 1, no. 2, pp. 55-60, 2007.

[4] J. Mork, and A. Mecozzi, Theory of non-degenerate four-wave mixing between pulses in a semiconductor waveguide, IEEE J. Quant. Elect., vol. 33, no. 4, pp. 545-555, 1997.

[5] J.M. Tang, and K.A. Shore, Characteristics of optical phase conjugation of picosecond pulses in semiconductor optical amplifiers, IEEE J. Quant. Elect., vol. 35, no. 7, pp. 1032-1040, 1999.

[6] K. Chan, C-K. Chan, L.K. Chen, F. Tong, Demonstration of 20-Gb/s all-optical XOR gate by four-wave mixing in semiconductor optical amplifier with RZ-DPSK modulated inputs, IEEE Photon. Tech. Lett., vol. 16, no. 3, 2004.

[7] H. Jang, S. Hur, Y. Kim, J. Jeong, Theoretical investigation of optical wavelength conversion techniques For DPSK modulation formats using FWM in SOAs and frequency comb in $10 \mathrm{~Gb} / \mathrm{s}$ transmission systems, IEEE J. Light. Tech., vol. 23, no. 9, 2005.

[8] A.M. Clarke, M.J. Connelly, P. Anandarajah, L.P. Barry, and D.A. Reid, Investigation of pulse pedestal and dynamic chirp formation on picosecond pulses after propagation through an SOA, IEEE Photon. Technol. Lett., vol. 17, no. 9, pp. 1800-1802, 2005.

[9] R. Trebino, K.W. Long, D. N. Fittinghoff, J. N. Sweetser, M. A. Krumbugel, and B. A. Richman, Measuring ultrashort laser pulses in the time-frequency domain using frequency resolved optical gating, Rev. Sci. Instrum., vol. 68, pp. 3277-3295, 1997.

[10] D.J. Kane, Real-time measurement of ultrashort laser pulses using principal component generalized projections, IEEE J. Sel. Top. Quant. Elect., vol. 4, no. 2, pp. 278-284, 1998.

[11] K. Ogawa and T.T. Lay, Self-phase modulation and spectral broadening of optical pulses in semiconductor laser amplifiers, IEEE J. Quantum Electron., vol. 25, no. 11, pp. 2297-2306, Nov. 1989.

[12] G. P. Agrawal and N. A. Olsson, Self-phase modulation and spectral broadening of optical pulses in semiconductor laser amplifiers, IEEE J. Quantum Electron., vol. 25, no. 11, pp. 2297-2306, Nov. 1989. 